\title{
Anxiety and Fear in Childhood Clinical and Critical Issues
}

\author{
Mohamad Q Abdullah* \\ Department of Clinical Psychology, University of Aleppo, Syria
}

*Corresponding author: Mohamad Q Abdullah, Department of Clinical psychology, Faculty of Education University of Aleppo, Syria, Email:mk.abdalah@yahoo.com

Submission: 械June 22, 2018; Published: 制 September 11, 2018

\section{Introduction}

Anxiety in childhood is problematic in many arenas. Social anxiety, one thought of as simply "shyness", does not more than cause a child to have difficulty with peer relations. When anxiety becomes overwhelming, a child will naturally avoid anxietyproducing stimulus. Hence, school refusal may begin. Children with anxiety are often preoccupied with worries about their success in activities and their ability to obtain the approval of others. These children may have persistent thoughts of self-doubt that they are unable control, and they constantly criticize themselves. Children may be preoccupied being on time to events and insist on doing a task "perfectly". In contrast to the ordinary, occasional worries or fear experienced in childhood, generalized anxiety, persist for at least six months and affects children throughout the day home, at school and with friends.

Anxiety defines a as "an emotion characterized by feelings of tension, worried thoughts and physical changes like increased blood pressure." It is important to know the difference between normal feelings of anxiety and an anxiety disorder that requires psychotherapy.

The clinical cues of anxiety are:

1. An emotional state.

2. Unpleasant.

3. Directed toward the future.

4. Intense tension and worry.

5. Somatic charges, especially construction of cheat.

6. Learned.

7. Have three components: Cognitive, Emotional/impulsive and physical reactions manifest bodily disturbances [1].

Anxiety symptoms and disorders are the number one health problem in the world, ranging from simple level to severe level such as panic disorder and social phobia and posttraumatic stress disorder. Although anxiety symptoms have been studied in adult population, anxiety disorders in children often are misjudged, despite them being very treatable conditions with good, persistent medical care. If anxiety in school children neglected and left untreated these anxiety symptoms in children likely progress to adult severe versions. Fear is an emotional response to accurate stimulus and situation threaten individual and accompanied with psychological and physical changes. This fear is normal and adaptive. If this fear is severe and irrational relating to accurate situation then being abnormal and is termed "phobia".

Fear and anxiety often occur together but these terms are not interchangeable. Even though symptoms typically overlap, a person's experience with these emotions differs based on their context. Fear relates to a known or understood threat, whereas anxiety follows from an unknown or poorly defined threat. It is very significant, for clinicians, to distinguish between adaptive/ normal fear and anxiety. We need to clarify these two terms that are closely intertwined. The following table summarized the most important differences: (Table 1) [2]. The main sources of fear and anxiety are the following: personality, past experiences, weather, stress, social activities, sleep, exercise, age, gender, temperament and parental treatment in childhood, cultural influences and school.

Table 1: The main sources of fear and anxiety are the following [2].

\begin{tabular}{|c|c|c|}
\hline Anxiety & Fear & Characteristics \\
\hline Unknown & Known & Object \\
\hline Internal & External & Stimulus \\
\hline Severe & simple & Grades \\
\hline Chronic & Acute & State \\
\hline Unconsciousness & Consciousness & Motivation of Response \\
\hline Defense mechanisms & Directed /Adaptive & Mechanisms \\
\hline intensive & absence & Conflicts \\
\hline
\end{tabular}

\section{Anxiety disorders in childhood}

The prevalence of clinical anxiety disorders in children is not known. While separation anxiety as a predictable phenomenon in the first year, as do multiple fears and simple phobias in later 
childhood, little is known currently about biological, familial, or experiential factors which might predict later periods of clinical distress with child anxiety disorders, and school avoidance. Several studies indicated a generally poorer prognosis for child anxiety disorders that occurs later into adolescence as contrasted with symptoms the first emerges with children in the 8-11 year-old range [3].

Anxiety is a subjective sense of worry, apprehension, fear and distress. Often its normal to have sensations on occasion, and so it is important to distinguish between normal levels of anxiety and pathologic levels of anxiety. The subjective experience of anxiety typically has two components: physical sensation (e.g., headache, nausea, sweating) and the emotions of nervousness and fear. Anxiety disorders, when severe, can affect a child's thinking, perceptions of environment, learning and concentration. It raises blood pressure and heart rate, and can cause a multitude of bodily components, such as vomiting, stomach pain, ulcers, diarrhoea, tingling, weakness, and shortness of breath, among other things.

\section{Types of Anxiety Disorders}
A. Generalized anxiety disorder
B. Obsessive-Compulsive Disorder
C. Post traumatic Stress Disorder:
1. Acute stress disorder
2. Panic Disorder
3. Social Phobia
4. Specific phobia
D. Adjustment Disorder with Anxiety
E. Anxiety Disorder due to a general Medical Condition
F. Drug-Induced Anxiety Disorder

\section{G. Anxiety Disorders Not Otherwise Specified}

As clinical/counselling psychologists and researchers, we are frequently confronting children who are having difficulties at school. Symptoms ranging from sporadic grades: poor test performance, intentional problems, decreased enjoyment and social activities.

\section{At school}

At school, the child with anxiety disorder may have a combination of symptoms listed below:

a. Excessive worry and anxiety about a variety of matters.

b. Repeated seeking of teacher approval.

c. An inability to explain the worries. Children may not understand what they are so anxious.

d. Inability to stop the worry. The worries continue.

e. Difficulty transitioning from home to school. Children may develop difficulty-entering school in the morning if they associate more worries at school. This may lead to late arrival times and tearful episodes at school.

Future investigations must undertake better definition and validation of assessment and diagnosis of anxiety disorders in children [4] that include not just child self-report, but family and teachers in schools with multi instruments such as: clinical interview, case study, rating scales, projective techniques for investigating the dynamic profile of personality.

\section{References}

1. Abdullah QM (2014) Psychopathology. University of Aleppo press, Syria.

2. Abdullah QM (2016) Introduction to mental health. Dar-Alfikr: Amman.

3. Casat DC (1988) Childhood anxiety disorders. Journal of Anxiety Disorders 2: 51-60.

4. Kazdin AL (1988) Child psychotherapy. Pergamon General psychology Series.

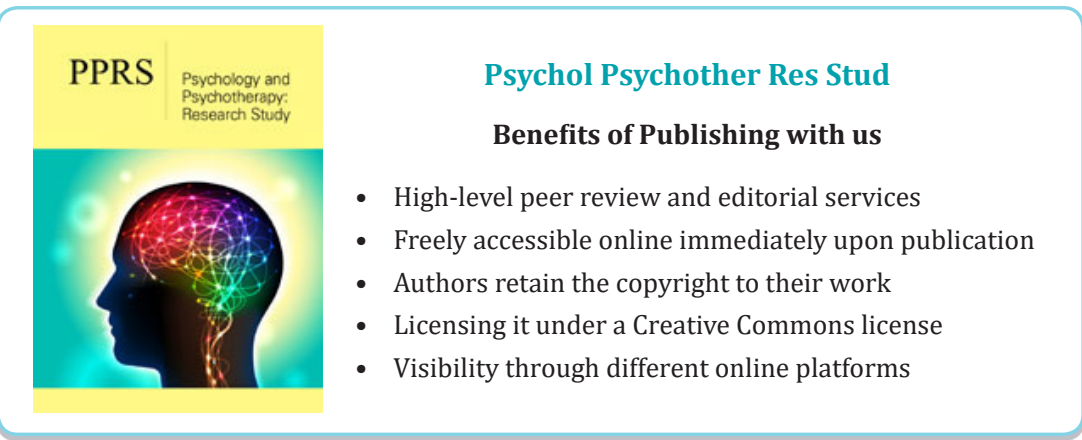

